\title{
Some Culturological Aspects of
} METI Problems with EM Radiation

\author{
Liliya Filippova and Vladimir Filippov \\ Russian Academy of Sciences Moscow
}

Correspondence | lnfilippova@yandex.ru; VVFilippov@yandex.ru

Citation | Filippova, Liliya, and Vladimir Filippov. 2020. "Some Culturological Aspects of METI Problems with EM Radiation." Journal of Big History IV (2): 128-135.

DOI | https://doi.org/10.22339/jbh.v4i2.4260

\begin{abstract}
Until the beginning of 2020, from 1974 to 2017, sixteen interstellar radio messages were sent to hypothetical extraterrestrial civilizations. They were sent from different radio telescopes and had different contents. The METI (Messaging Extraterrestrial Intelligence) projects had different concepts, some of which seem highly controversial. Therefore, a short list of criteria for future METI projects is proposed. As an example, we suggest an interstellar message in the radio frequency range of the electromagnetic spectrum that we call "Golden Wings of a Lemniscate" (GWL). The GWL project is a simple message from Earthlings about their intelligence and readiness for interstellar contact. The digital part of the message contains ten images of lemniscates. The right half of the lemniscate includes the Golden Ratio concept with graphical drawings; the left parts of the lemniscate remain empty for ET reply messages. The experience of developing new projects of interstellar messages, initiative or response, is valuable. In the process of this work, various aspects of METI will be investigated: scientific, technical, informational, cultural, ethical, educational, sociological, psychological, historical, etc.
\end{abstract}

\section{SETI}

In April 1960, at the Green Bank National Radio Astronomy Observatory in the USA, the young 30-year-old scientist Frank Drake organized the first search for radio signals from extraterrestrial civilizations. A radio telescope with an antenna diameter of 85 feet $(26$ meters) was used in observations; its receiver was tuned to a frequency of $1420 \mathrm{MHz}$ (a radio emission of interstellar hydrogen at a wavelength of $21 \mathrm{~cm}$ ). Two close stars were chosen for radio listening: solar-type Tau Ceti and Epsilon Eridani, which are located at dis- tances of 12 and 10.5 light-years, respectively.

Observations continued in May, June, and July. The total observation time of these two stars was two hundred hours; however, the pioneering search for signals from extraterrestrial civilizations by Drake forever entered the scientific history of humankind. Signals of extraterrestrial origin were not detected

(Drake 1960). As of the current date (14 March 2020), with the help of ground and space telescopes, over 4214 planets have been discovered near stars in all the constellations of the sky and dis- coveries continue (Exoplanet.eu). The star Tau Ceti has four planets discovered. The star Epsilon Eridani has one planet discovered and a second one awaiting confirmation.

Diversified research has begun on the problem of the search for extraterrestrial intelligence in scientific centers of many countries, including international cooperation. Later, this area of activity received the term SETI (Search for Extraterrestrial Intelligence). Over the past decades, SETI strategies have been developed to use high-tech telescopes 
in the accessible ranges of electromagnetic radiation.

An impressive example is a project for the search for extraterrestrial intelligence implemented by the SETI Berkeley Research Center in the US, in the radio and optical bands, which launched in 2016 (Breakthrough Listen). The Green Bank (GBT) radio telescope, the Parkes radio telescope in Australia, and the automated Planet Finder (an optical robot telescope equipped with ultramodern spectrographic technology) are used for observations.

GBT (USA) is the largest fully-steerable radio telescope on Earth with a giant parabolic antenna of one hundred meters (328 ft.) in diameter. The Australian Parkes radio telescope is also fully rotated with a parabolic antenna of sixty-four meters (210 ft.) in diameter. SETI Berkeley Center began to connect partners from different countries to the Breakthrough Listen Project on radio telescopes: MeerKAT (South Africa), NenuFAR (France), FAST (the world's largest radio telescope in China), MWA (Western Australia), LOFAR stations in Ireland and Sweden, Jodrell Bank (England), e-MERLIN (seven radio telescopes throughout the UK), Sardinia (Italy) and Allen Array (SETI Institute in the USA), as well as VERITAS (gamma-ray telescope in the USA) and Keck Optical Observatory (Hawaii, USA) (Breakthrough Listen).

The Breakthrough Listen program includes an examination of 1,00o,ooo selected stars, the center of our Galaxy and the entire galactic plane. Outside the Milky Way, interstellar communications are being searched for from the one hundred galaxies closest to us. The Breakthrough Listen initiative will span ten years, for which sponsor Yuri Milner has allocated a total of \$US 100 million. In 2019, the SETI Berkeley Center established further collaboration with scientists working on the TESS (Transiting Exoplanet Survey Satellite) space optical telescope mission. The TESS satellite telescope entered low Earth orbit in April 2018 to search for planets orbiting bright, relatively close stars. In the TESS program, the study of 200,ooo stars of the solar-type and red dwarf stars, at distances up to $\sim 650$ light-years, scattered throughout the starry sky (TESS). All observational data for the Listen project, combined with new planet discoveries using TESS, are analyzed in the SETI plan for the detection of so-called "techno-signatures," a term proposed by Jill Tarter of the SETI Institute to refer to the technological activities of extraterrestrial civilizations found in Breakthrough Listen observations. This may be a "leak" from the planet from the transmissions EM range, radiation from alien radars, or laser communications and other technogenic activities in the area of their habitat.

In Russia, according to the SETI program, observations were conducted from the second half of the 1990s (to search for both "technological leakage" and possible signals) using different telescopes, the largest of which are the RATAN-6oo radio telescope and the BTA optical telescope. The RATAN-6oo radio telescope has a ring antenna with a diameter of six hundred meters (1889 $\mathrm{ft}$.) and the optical Azimuthal Large Telescope (BTA) with a mirror diameter of six meters are located in the North Caucasus and belong to the Special Astrophysical Observatory of the Russian Academy of Sciences. Some noteworthy results require additional observations on large radio telescopes (Chuprikov and Filippova 2007; Rudnitskiy 2016). It should be noted that for several reasons, starting from 1960 , there is not a single example of continuous multiyear monitoring of any stars or space objects selected by justified astronomical criteria for SETI in the search programs for technological civilizations. Before the alleged discovery of "technosignature civilizations" or a higher level of development (emerging from the "techno-signature" phase) using high planetary and space technologies, questions arise:

1) Do all technological civilizations profess the position of "information silence" in all ranges of the electromagnetic spectrum to avoid the risk of contact with other civilizations? How can this hypothetical fact, if confirmed in long-term observations, affect the policy of mankind in terms of interstellar communication: to remain silent or to broadcast?

2) Are there space civilizations sending interstellar messages to transfer the interstellar communication initiative to their future generations? In this case, what should be the strategy of terrestrial civilization for terrestrial and space SETI monitoring for "random reception" or to reveal a "convergent space schedule" for receiving repeated or new extraterrestrial messages?

\section{METI}

On November 16, 1974, at the ceremony dedicated to the reconstruction of the Arecibo 30o-meter radio telescope in Puerto Rico, for the first time, a short radio message was sent from Earth to extraterrestrial civilizations. At the speed of light on a radio wave of 12.6 $\mathrm{cm}$, the encoded "Arecibo Message" rushes to the globular star cluster M13 in Hercules and will arrive at the target in twenty-five thousand years. Its content and principles of coding and 
decoding were developed by the astronomer "SETI Pioneers" Frank Drake and Carl Sagan with the participation of colleagues (Steele 1999). From 1974 to 2017, sixteen interstellar radio messages were sent. Among them, in addition to Arecibo Message (1974), there were fifteen radio messages to twenty-four stars. Messages of different contents were sent to four stars: 37 Geminorum (37 Gem), 47 Ursae Majoris (47 UMa), 55 Cancri ( $55 \mathrm{Cnc}$ ), and Gliese 581 in the Libra constellation. The record-holder is star 55 Cancri, to which radio messages from the Earth were sent: in 2003 (Cosmic Call 2 from Yevpatoriya, Crimea); in 2012 ("Wow! Reply" from Arecibo, USA); in 2013 (JAXA Space Camp (UDSC-1) and 2014 (JAXA Space Camp (UDSC-2) from Japan.

It is interesting to note that the same content messages were sent to different stars in the projects: Cosmic Call 1 (1999) was sent to four stars, Teen Age Message (2001) was sent to six stars, Cosmic Call 2 (2003) was sent to five stars, "RuBisCo Star" (2009) was sent to three stars, and "Wow! Reply"(2012) was sent to three stars. The list of completed Projects from 1974 to 2017 is most fully represented on Wikipedia in English. Russian scientist Dr. A. Zaitsev proposed the abbreviation METI (Messaging to Extraterrestrial Intelligence), which was strengthened in scientific everyday life. Zaitsev was the scientific and technical leader of four Projects of interstellar radio messages from the Evpatoria space center: in 1999, Cosmic Callı to four sun-like stars; in 2001, "Teen Age Message" to six sun-like stars; Cosmic Call 2 in 2003 to three sun-like stars and two red dwarfs; in 2008 "A Message from Earth" to the star Gliese 581, a red dwarf in the constellation of Libra, with three confirmed planets at the time of sending.
The messages were designed with a noise-resistant format and characters. Its main characteristics are as follows: the central frequency is $5.01 \mathrm{GHz}$ (6 $\mathrm{cm}$ ), and the effective area of the transmitting antenna is about 2500 square meters. The work is conducted from a highly stable hydrogen generator in a mode of continuous coherent radiation with average capacity up to $150 \mathrm{~kW}$. The digital information is transmitted using carrying frequency manipulation with a deviation of $48 \mathrm{kHz}$ : the nominal shift of $+24 \mathrm{kHz}$ corresponds to the symbol "1," and the shift of $-24 \mathrm{kHz}$ corresponds to the symbol "o" (Braastad and Zaitsev 2003). Interstellar messages from the Earth were sent from different radio telescopes at different wavelengths, have different transmission durations, have different contents, and went to stars with no proof of the existence of technological civilizations, and it is still unknown whether some of the target stars have planets.

Here we would like to propose a short list of criteria for future METI projects:

1) Have understandable content for the Earth's scientific and cultural community. In the case of using any special language representing the content messages for extraterrestrial civilization, it should be presented to the earthly public in understandable ways - in drawings or diagrams and in the project description.

2) Be peaceful. The introduction of potentially malicious code into any METI projects should be excluded.

3) The content should be interesting for project developers and the cultural Earth community.

4) It should also be creative and aesthetic. Each project must have intellectual and emotional value for the
METI story. Include some elements of beauty from an earthly point of view.

5) Interstellar messages to extraterrestrial civilizations should be considered as messages from people of METI communities, and not as a "Message from all of Humanity on Earth." Modern humanity belongs to METI in different ways. Some approve of this activity, yet others are opposed to METI and even SETI. It will probably be so in the future. Therefore, it is not correct to consider interstellar messages as messages from all of humanity because these are messages to extraterrestrial civilizations solely from people from specific METI communities.

6) Do not accompany the interstellar message from Earth with ballast information that is not accessible for decipherment by civilization, by the Recipient (names and short texts of the project participants who provide material support to the project, or any advertising messages).

This last recommendation requires explanation. For example, the Cosmic Call 1999 interstellar radio message consisted of a scientific part and 50,00o short messages from people who made a monetary contribution to the project. After conversion to binary stream, the total length of the Evpatoria message exceeded 1.7 million bits. Zaitsev said in an interview "Any earthling could take part in the 2003 message, having paid $\$ 15$ to the organizers. Therefore, in addition to the scientific part, the cosmic call sent common communications such as "Hello, aliens! Send me a meteorite made of gold," and "I love my cat Buck." In reality, only the scientific part of the message went to the stars. The transmission power of "commercial announcements" was such that it was possible to try to accept them only no 
farther than the orbit of Jupiter. At a greater distance, they merged with radio noise. (Astroforum), but minisponsors believed that their names and texts would reach the stars.

Similarly, on June 12, 2008, the radar of the EISCAT Space Center in Svalbard broadcast a coded advertisement of Doritos brand tortilla chips on a frequency (500 MHz). The ad was sent to hypothetical residents of the star 47 UMa, 46 light-years distant from the constellation Ursa Major. On August 28, 2009, a NASA/CSIRO seventy-meter long-range space communications antenna near Canberra, Australia, broadcast a four cm wavelength (7.145 MHz) in the direction of the nearby red dwarf Gliese 581 from HELLO FROM EARTH (HFE). This message contains 25,878 short messages from people from ten countries and has a volume of 2,845,345 bits. Here are some examples of messages from Earthlings (The best messages from Canberra (2009)):

“Hello, Gliese 581d inhabitant. Can you help us humans travel through space and become smart like you? Please do not eat us we are a friendly race.” Angus Pigott; Canberra, Australia

"If you know the meaning of life, please send it to us. If not, let's celebrate together anyway! Thanks in advance, from a curious carbon-based life form!" Monica Echagüen; Barcelona, Spain.

"Hello from Varun on Earth. I hope I can meet you someday and we could become friends. My father thinks our cats are aliens-maybe they came from your planet!" Varun; Sydney, Australia.

"We Are A Smart Race Of People. We Hope To Find More In The Cosmos We Hope You Are Peace Loving Crea- tures. Let's Share Our Histories. See You In The Future." Jordan; Canberra, Australia.

Future communications from the Earth should be meaningful and worthy of content not only for aliens but also for future generations of Earthlings. This is the ethical aspect of the culture of terrestrial civilization. "The notions of morals and good are universal, like the Pythagoras theorem. Civilizations do not survive if they behave otherwise." (Russian "Pioneer of the SETI" academician N. S. Kardashev).

Everyone can participate in the development of projects for future interstellar radio messages, regardless of professional interests, nationality, religion, etc. Those who wish to participate in METI projects send their application and their project to the future international scientific and cultural center METI. Currently, The IAA SETI Permanent Committee does not support METI due to reasonable risks to humanity. However, it cannot be ruled out that in the future there will be support for the creation of the METI Center to study various problems of interstellar communication with extraterrestrial civilizations. Those wishing to participate in the project indicate their field of activity in the application: content development, technical assistance, coding, choice of target stars, etc.

New METI projects should be made available to the public on the Internet for review and determination of public interest. The best ideas, original proposals, and new projects would then be considered by the METI International Scientific Council for possible implementation. Financial support for future METI projects for sending them to selected stars should be welcomed. However, financial support should not entitle donors to include the name and per- sonal text in the information stream attached to the main METI content. Gratitude for financial support can be a certificate with the name of the philanthropist and a note that his or her name and text (if any) will be registered in the Thanks Catalog of the METI Center.

The METI wiki portal should contain catalogs with detailed information about each interstellar message. In the case of broadcast in the electromagnetic range, of interest are

location of the transmitting system, data about the telescope: transmitter power, and electromagnetic transmission range; date and time of broadcasting, duration of broadcasting; and message size in bits and bit rate; description of the contents of the message and its visual representation; encoding method and decoding options; estimated equatorial coordinates of the target star for aiming the transmitting system on the date of arrival of the message to it; data about the star and its planetary system; the estimated arrival epoch of METI, and the estimated power of the signals in the star system; names and other data of developers of the contents of the message and scientific and technical personnel providing preparation and broadcasting; project cost and financial support received from sponsors; photos, videos, etc. Now there is no such detailed information about METI projects that were already sent to the stars. Future METI projects may have different concepts, such as a message about humanity and some of its cognitive achievements from the field of science and culture, selected works of art or music, or a variety of composite structures (e.g., call signs, music, digital parts), or the message could be simply about the intelligence of senders and their readiness for interstellar communication. 
Project of the Content of the Interstellar Radio Message "Golden

\section{Wings of a Lemniscate"}

Before the scientific discovery of extraterrestrial civilization, the authors are proposing a project of the interstellar radio message "Golden Wings of a Lemniscate" (GWL) first presented in a report at the symposium "Horizons of Astronomy and SETI" in SAO RAS (2005) and published in the collection of articles of this symposium (Filippov et al, 2007). Here we present an updated version of the GWL project with several new elements.

The fundamental idea of the GWL project is simply a message about the intelligence of the inhabitants of the third planet of the sender's star and the readiness for interstellar contact. An intermediary language for the messages in this concept is informational drawings without specific information about the solar system, the Earth or humanity.

For this project, from the huge number of pictorial symbols in the history of the culture of mankind (art and mathematics), the lemniscate of Bernoulli was chosen. The lemniscate is the mathematical symbol for "infinity," which also symbolizes the vastness of outer space. Using manipulations (variations) of one of the parameters of the carrier radio wave, one can transmit a sequence of binary symbols that can be decomposed into a rectangular matrix of primes containing an image of a lemniscate with a picture. Prime numbers for the matrix are selected such that their ratio is close to the "golden number" $=1.618$.

The golden ratio reflects the emotional perception of harmony in a person of the Earth and, perhaps, is universal knowledge for "space subjects" of various types (Lefebvre and Efremov 1999), including space systems with artificial intelligence (AI). The proposed interstellar radio message can become the bearer of the idea of the universality of harmony in the universe, known to the senders of the message (Earthlings) as the "golden section," and to "speak with pictures" placed in the lemniscate. The main content of the interstellar radio message will be preceded by the transmission of a repeating series of "digital call signs" - images of small lemniscates in a small "golden" rectangular scan, including the repetition of their negative displays (white inverted to black) for a "hint." To transmit the call sign, coherent signal broadcasting in time intervals in the proportion of the golden ratio with the radiation duration, for example, 157, 97, 97, 6o seconds, respectively, with pauses, can be used. The presence in the call sign of primes and their ratio $\sim 1.62$ is intended to be a hint for searching for the compilation of matrices based on them, which should help decipher the content of the digital part of the radio message. Call signs can be broadcast using the "relative signal transmission methods" proposed by Professor N. T. Petrovich for interstellar radio communications. (Petrovich 1986; Filippov et al. 2005).

After the completion of the digital call sign transmission, the transmission of the main message content, which contains ten different elements, begins. The number of elements is not accidental; it is intended to lead the message recipients to a hunch about the significance of the decimal system of calculus for its senders. All ten elements contain identical images of Bernoulli lemniscates in the right "wing" of which there are drawings; the left is left blank for an answer. In the "golden" rectangles above, the numbers of the natural series from 1 to 10 are inscribed, and the notion of the Earth designation of each figure by serial numbers is given below. The elements of the message intentionally include images of simple geometric figures (segments, triangle, circle, square, spiral, etc.), which should be familiar and recognizable by ET. Note that the selected geometric figures are associated with the history of the development of natural science on Earth and with the names of prominent philosophers and mathematicians who studied them. Any choice of designs for METI will be controversial. The rationale for our choice is briefly made in the comments to the drawings.

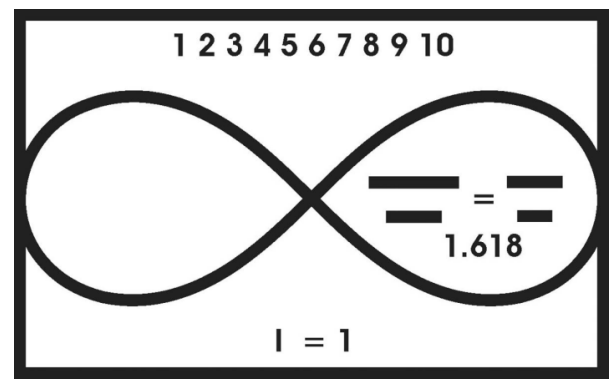

Figure 1. Sections in proportion to the golden ratio

If the ET, by analyzing this figure, guess to measure the lengths of large segments of the left and right parts, separated by the equal sign, and divide them into smaller lengths, then they will receive the value of the "golden number" in their calculus and will be able to see the Earth spelling of the golden number 1.618 .

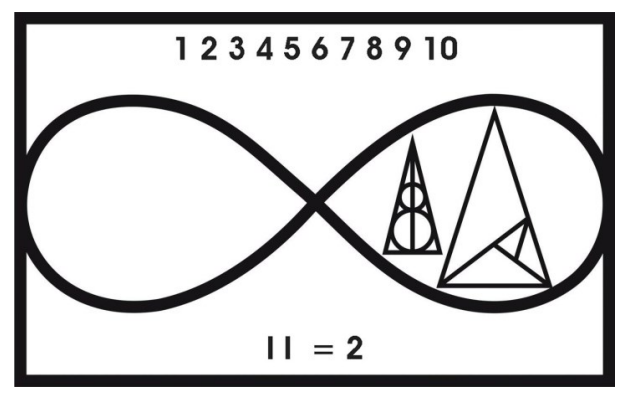

Figure 2. Golden Triangle

The second element in the language of geometry offers ET to compare their 
concepts of a triangle, a circle, and its diameter, in the proportions of the golden section.

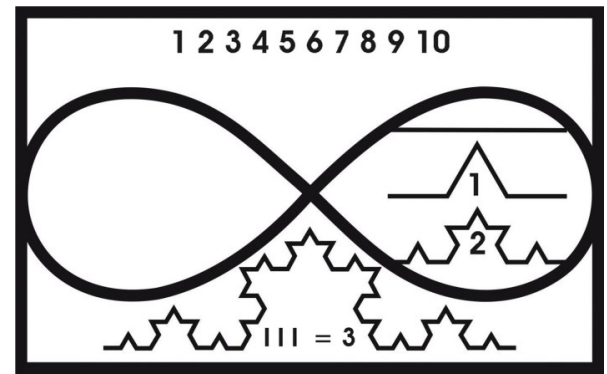

Figure 3. Fractal Koch curve

The term "fractal" was proposed by Benoit Mandelbrot in 1975 to refer to selfsimilar sets with fractional dimensions. The fractal repeats itself at different scale levels. Of the geometric fractals, the most famous is the Koch snowflake, which is based on an equilateral triangle. This fractal is named after the Swedish mathematician Helge von Koch (1870-1924), who first described it in 1904. The main arguments for including the Koch fractal are its simplicity for coding and its beauty. The lemniscate presents a part of the fractal Koch curve in three iterations.

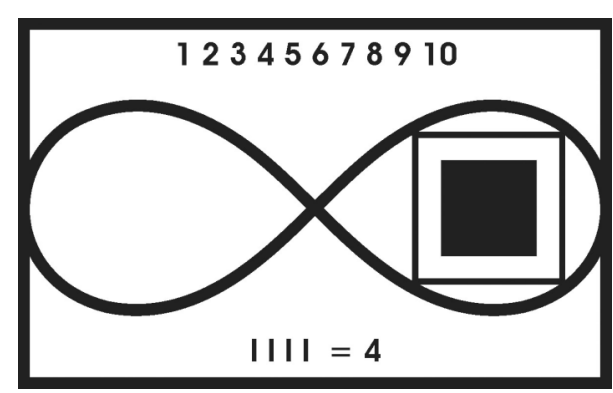

Figure 4. The black square of Malevich
Kazimir Malevich (1878-1935). This is one of the most discussed and most famous paintings in Russian art. Malevich's Black Square suggests the inclusion of his image into the lemniscate. The square is not a problem for coding and, in our opinion, is a good example of "rationality" of the signal.

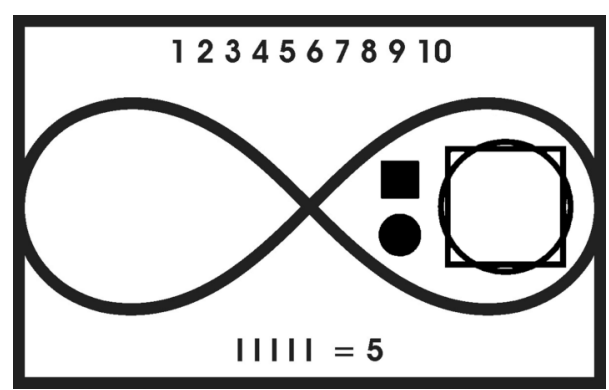

Figure 5. Squaring a circle

This is the name of the famous task: using a compass and a ruler to build a square, equal in area to a circle of a given radius. The inclusion of the "quadrature of the circle" picture has a historical aspect (for terrestrial civilization) and a test of "reasonableness" for hypothetical stellar message Recipients. Representatives of extraterrestrial intelligence are invited to think about the task from this picture.

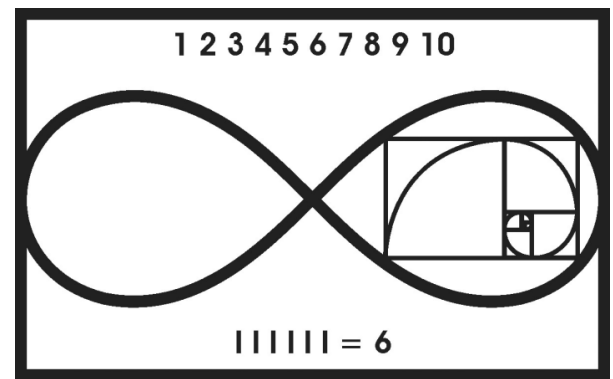

The square is known in many ancient cultures; for the Pythagorean School, the square was an emblem of divine balance, expressed geometrically. "Black Suprematist Square" is a picture created in 1915 by avant-garde artist
Figure 6. Logarithmic Golden Spiral

The sixth element of the project contains two wonderful curves: a lemniscate and a logarithmic spiral. A special type of logarithmic spiral is the golden spiral, so-called the Fibonacci Spiral. In the culturological aspect, the lemniscate and the logarithmic spiral are Earthly symbols of infinity and the evolution of life and mind.

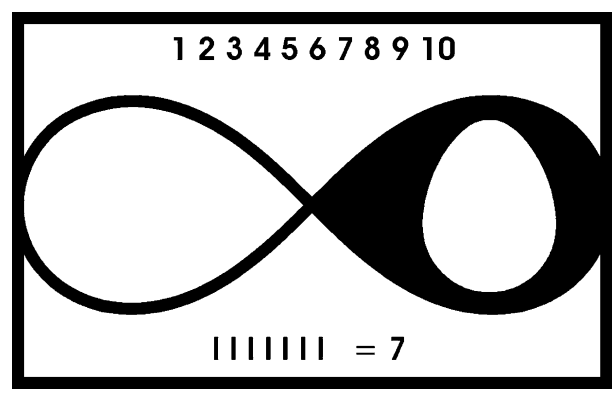

Figure 7. Egg in Golden Ratio Proportions

It is proposed to include into the lemniscate of the seventh element a symbolic image of an egg constructed in the proportions of the golden section. In different epochs of life, oviparous animals existed on Earth. Currently, their representatives are birds, fish, reptiles, marine animals, insects, and mammals from the families of platypus and echidna. It has been found that in many birds the egg shape has a golden ratio. The egg symbol is used in architecture, design, sculpture, jewelry. Huge eggs on the towers and the cornices adorn the wall of the theater and museum of the great artist Salvador Dali (19041989) in Spain; this is one of the most popular surreal buildings in the world. Russian jeweler Peter Carl Faberge (1846-1920) created the famous collection of Faberge eggs. The series was created between 1885 and 1917 for the Russian imperial family and private buyers. The idea of a "world (cosmological) egg" inspires many artists to work, among them Russian artist and astronomer, Nina Yakimova, who has created more than one hundred fifty collages on this subject. 


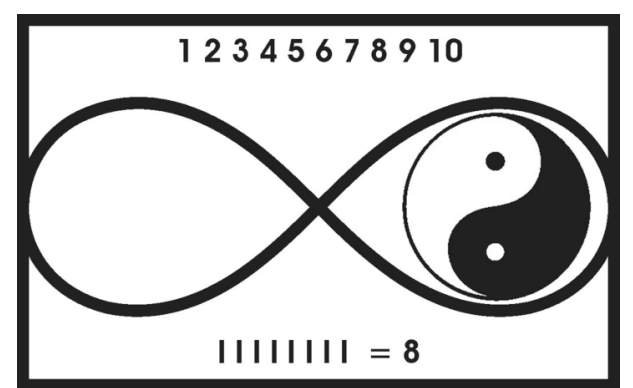

Figure 8. Yin Yang Symbol

Yin-Yang symbolizes that everything in the universe is in motion, changing, and has its opposite.

The Yin-Yang symbol included in the content of the GWL message will show a long-valued element of the culture of a huge part of humanity on Earth.

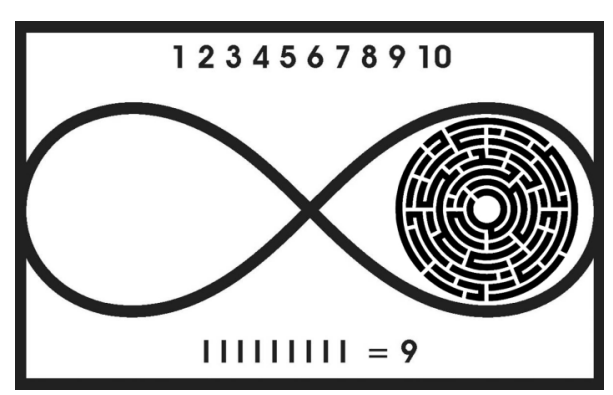

Figure 9. Labyrinth

The labyrinth suggests finding a way out of the dead ends in this image. This is a game message and a simple intelligence test.

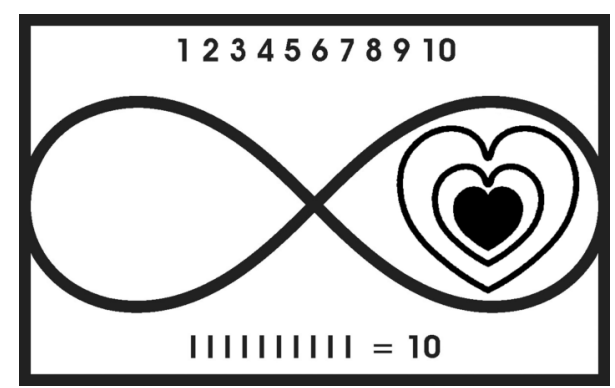

Figure 10. Heart
The popular drawing of the heart is a symbol of love, spiritual harmony, and happiness. Despite the amazing simplicity of its outlines, this symbol remains one of the strongest positive emblems of the world. This GWL lemniscate has a symbolic drawing of three hearts representing the hearts of father, mother and child in people and a huge number of animals. The images of hearts are based on the golden spiral.

The meaning of this picture may not be understood when deciphering the image, but for Earthly senders, it is an important symbol of love and hope for a positive response of intelligent interstellar recipients of the GWL message.

\section{Conclusion}

It should be noted that to detect any of the sent interstellar radio messages from Earth, it is necessary that there exists in the systems of destination stars technologically advanced civilizations; that ET's telescopes are used to search for transmissions from space civilizations in different ranges of the electromagnetic spectrum; and that the direction of our solar system is included in monitoring in the ranges of the sent messages. Therefore, before the discovery of extraterrestrial civilizations, the sending of interstellar messages into space is unlikely to be a success at the reception. Is it necessary to develop the content of new messages from the Earth, initiative or response, in case of detection and decryption of messages from extraterrestrial civilizations? The experience of developing new interstellar message projects seems valuable. In the process of this work, various aspects of METI will be investigated, including but not limited to scientific, technical, informational, cultural, ethical, educational, sociological, psychological, and historical. This will help new generations of mankind realize the prospects of establishing interstellar communication with one or more extraterrestrial civilizations if their discovery will take place.

\section{References}

"Active SETI: Transmissions: Stars to Which Messages Were Sent." Wikipedia: The Free Encyclopedia. https:// en.wikipedia.org/wiki/Active_SETI.

Barclay, Thomas, ed. TESS Science Support Center. NASA. https:// heasarc.gsfc.nasa.gov/docs/tess/.

Braastad, Richard and Alexander Zaitsev. "Synthesis and Transmission of 'Cosmic Call 2003' Interstellar Radio Message." http://www.cplire.ru/ html/ra\&sr/irm/CosmicCall-2003/ index.html.

Breakthrough Listen at UC Berkeley. https://seti.berkeley.edu/listen/.

"Breakthrough Listen to Collaborate with Scientists from NASA's Transiting Exoplanet Survey Satellite (TESS) Team to Look for Signs of Advanced Extraterrestrial Life.". Breakthrough Initiatives. October 23, 2019. https:// breakthroughinitiatives.org/news/27

Catalog. Exoplanet.eu. Accessed March 14, 2020. http://exoplanet.eu/ catalog/.

Chuprikov A. A. and L. N. Filippova L.N. "Observations of SETI Objects on the Radio Interferometric Network of the ASC LPI." Special Bulletin of the SAO RAS 2007: 60-61, 208-211. SAO RAS, 2007. In Russian. http:// wo.sao.ru/Doc-k8/Science/Public/ Bulletin/Vol6o-61/p2o8.pdf

Drake, F. D. "Project OZMA: The Search for Extraterrestrial Intelligence." Proceedings of the NRAO Workshop No. 11. May 20-22, 1985. Eds. K. I. Kellermann and G. A. Seielstad. p. 17. Refer to Drake, F. D. 1960. Sky \& Telescope 39, 140. https:// 
technosearch.seti.org/list/projectozma. FULL PAPER.

Filippov V. V., L. N. Filippova, N. T. Petrovich and E. V. Ulitina. "The Project of a Radio Message for Extraterrestrial Civilizations: 'Golden Wings of a Lemniscate'." Special Bulletin of the SAO RAS 2007: 60-61, 239-247. SAO RAS, 2007. In Russian. http:// wo.sao.ru/Doc-k8/Science/Public/ Bulletin/Vol6o-61/p239.pdf.

Filippov V. V., N. T. Petrovich and L. N. Filippova. 2005. "The Project of the Interstellar Radio Message: "Golden Wings Lemniscates: Increasing the Noise Immunity and Range of Earth Signals by Relative Methods and by Repeating Elementary Premises for Their Detection in Noise. Publications NTORES named after A. S. Popov. LX:2. Moscow. In Russian. http://www.autex.spb.su/download/ dsp/dspa/ntores2005/t-21.pdf.

Filippova L. N. "Radio Searches of Extraterrestrial Civilizations.” SCC SETI Newsletter. Bulletin of SETI No. SCC SETI 23/40. In Russian. http:// lnfmi.sai.msu.ru/SETI/koi/ bulletin/4o/soder.html.

"Horizons of Astronomy and SETI". Conference: "SETI-2005" topic. Astroforum.

Lefebvre, V. A., and Yu N. Efremov. "Possible Analogues of Cognitive Processes in the Patterns of X-ray Variability of the Rapid Burster." Astronomical and Astrophysical Transactions 1999:18 (2) 355-342.

Rudnitskiy, G. M. 2016. "Search for Signals of Extraterrestrial Civilizations." Odessa Astronomical Calendar. 2017: 18, 157-165. Odessa, Ukraine: Astroprint. In Russian.
Steele, Bill. It's the 25th Anniversary of Earth's First (and Only) Attempt to Phone E.T." Cornell News. November 12, 1999. Archived from the original on August 2, 2008. Retrieved March 29, 2008. https://web.archive.org/ web/2008o802005337/http:// www.news.cornell.edu/releases/ Nov99/Arecibo.message.ws.html.

TESS. MIT-TESS. https://tess.mit.edu/.

University of Leicester. "Doritos Makes History With World's First Extra Terrestrial Advert." SpaceRef. June 28, 2008. http://spaceref.com/news/ viewpr.html?pid=25814. 